\title{
Photometric studies of two solar type marginal contact binaries in the Small Magellanic Cloud
}

\author{
Devarapalli Shanti Priya*, Jagirdar Rukmini ${ }^{\dagger}$
}

Department of Astronomy, Osmania University, Hyderabad, Telangana - 500007

\begin{abstract}
Using the Optical Gravitational Lensing Experiment catalogue, two contact binaries were studied using data in the $\mathrm{V}$ and $\mathrm{I}$ bands. The photometric solutions for the $\mathrm{V}$ and $\mathrm{I}$ bands are presented for two contact binaries OGLE 003835.24-735413.2 (V1) and OGLE 004619.65-725056.2 (V2) in Small Maglellanic Cloud. The presented light curves are analyzed using the Wilson-Devinney code. The results show that the variables are in good thermal and marginal geometrical contact with features like the O'Connell effect in V1. The absolute dimensions are estimated and its dynamical evolution is inferred. They tend to be solar type marginal contact binaries. The 3.6-m Devasthal Optical Telescope and the 4.0-m International Liquid Mirror Telescope of the Aryabhatta Research Institute of Observational Sciences (ARIES, Nainithal) can facilitate the continuous monitoring of such kind of objects which will help in finding the reasons behind their period changes and their impact on the evolution of the clusters.
\end{abstract}

\section{Introduction}

The Small Magellanic Cloud (SMC) is classified as an irregular dwarf galaxy with coordinates $\alpha_{2000}=$ $00 \mathrm{~h} 52 \mathrm{~m} 44.8 \mathrm{~s}, \delta_{2000}=-72^{\circ} 49^{\prime} 43^{\prime \prime}$. A large number of clusters and variable stars were discovered in the SMC through microlensing search projects such as the massive compact halo objects (MACHOs), the Earth Resources Observation System (EROS), and the Optical Gravitational Lensing Experiment (OGLE). OGLE II (Udalski et al. 1997, 1998; Wyrzykowski et al. 2003) has provided a catalog of B, V and I magnitudes for 1350 eclipsing binaries in SMC fields and has classified 733 stars as EA, 570 as EB and 150 as EW type binaries. Studies of these types of eclipsing binaries are essential because of their short periods $(<1 \mathrm{~d})$ and mass transfer that take place between the components. They provide an insight to the evolutionary state of the stars. Among the eclipsing binaries in the SMC, two short period solar type contact binaries were chosen for the current study, as they tend to be the most interesting objects in the evolutionary scenario. Models of contact binaries have been discussed by many investigators (e.g. Lucy 1968, Mochnacki 1981, Yakut \& Eggleton 2005, Stepien 2006).

\section{Data selection and Analysis}

The variable stars for present work were chosen based on the following selection criteria: an orbital period of at maximum 0.4 days, a temperature similar to solar analogues, an I-passband light curve

*astroshanti@gmail.com

${ }^{\dagger}$ rukminiouastro@yahoo.com 
Table 1: Photometric parameters of the selected systems.

\begin{tabular}{ccccc}
\hline OGLE ID (Variable ID) & Period (d) & I (mag) & B-V (mag) & Temperature (K) \\
\hline OGLE J003835.24-735413.2 (V1) & 0.26909 & 14.85 & 0.97 & 4725 \\
OGLE J004619.65-725056.2 (V2) & 0.37663 & 12.71 & 0.64 & 5850 \\
\hline
\end{tabular}
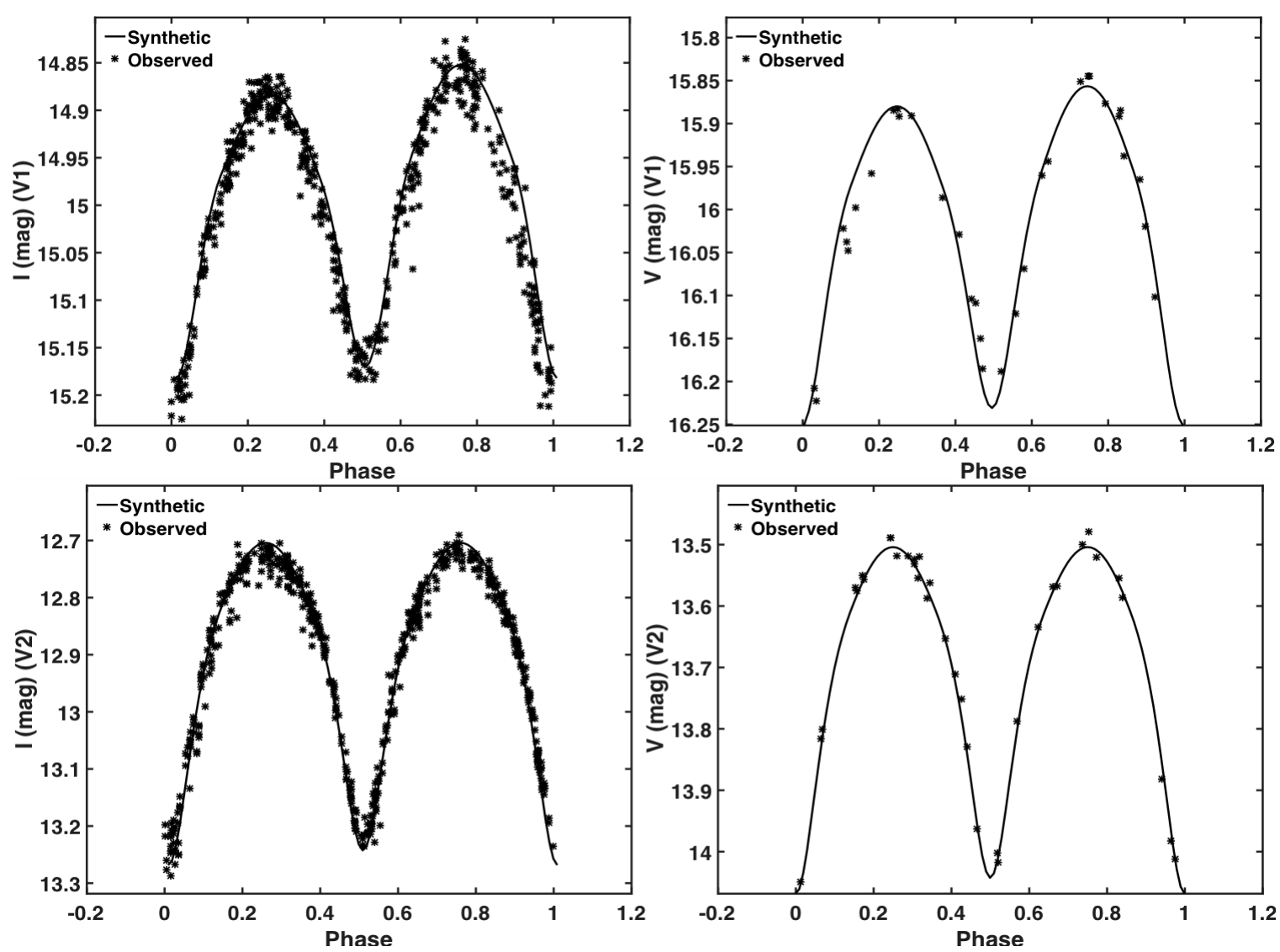

Figure 1: Observed and synthetic light curves in I (left) and V (right) bands. Equations (1) and (2) were used for the calculation of the phase.

amplitude of about 0.4 mag with a scatter of less than 0.05 mag, and data showing a complete phase coverage in the light curve. The data in the $\mathrm{V}$ and I passband observed through the OGLE II survey were chosen for a photometric analysis and the parameters were obtained using these data. The details of the variables are tabulated in Table $1^{1}$.

To obtain reliable results, the effective temperature of the primary stars are derived from the B-V values using Allen's (2000) table (see temperature values in Table 1). Initially, the effective temperature of the secondary component $\left(\mathrm{T}_{e, c}\right)$ was assumed to be equal to primary component's temperature $\left(\mathrm{T}_{e, h}\right)$. In order to determine the basic parameters of the two variables, the 2003 version of the differential correction (DC) program (Wilson \& Devinney 1971; Van Hamme \& Wilson 2003) was used. The period and times of minima of both the variables were determined using the Period04 package (Lenz \& Breger, 2005) and the Kwee \& van Woerden method (1956), respectively. The ephemerides (in the form: Min I $=\mathrm{HJD}$ (error) + period(error) E, where E is Epoch number) obtained for V1 (Eq. (1)) and V2 (Eq. (2)) using the I band data are:

$$
\text { MinI }=2450622.038881(15)+0^{d} .26909(3) E
$$


Table 2: The photometric parameters obtained for OGLE 003835.24-735413.2 (V1) and OGLE 004619.65-725056.2 (V2) using the Wilson-Devinney method.

\begin{tabular}{|c|c|c|c|c|c|}
\hline & \multicolumn{2}{|c|}{ OGLE 003835.24-735413.2 } & \multicolumn{2}{|c|}{ OGLE 004619.65-725056.2 } \\
\hline & & I & V & I & V \\
\hline Period (d) & & 0.2690 & 0.2690 & 0.3766 & 0.3766 \\
\hline $\mathrm{T}_{e, h}(\mathrm{~K})$ & & 4725 & 4725 & 5850 & 5850 \\
\hline $\mathrm{T}_{e, c}(\mathrm{~K})$ & & $4470 \pm 31$ & $4509 \pm 22$ & $5718 \pm 66$ & $5734 \pm 32$ \\
\hline $\mathrm{q}$ & & $1.30 \pm 0.03$ & $1.37 \pm 0.05$ & $0.81 \pm 0.05$ & $0.91 \pm 0.04$ \\
\hline $\mathrm{i}^{o}$ & & $63.09 \pm 0.40$ & $66.21 \pm 0.34$ & $73.69 \pm 0.32$ & $73.66 \pm 0.32$ \\
\hline$\Omega$ & & $4.2783 \pm 0.0689$ & $4.3341 \pm 0.066$ & $3.4782 \pm 0.0471$ & $3.6845 \pm 0.0770$ \\
\hline fill-out factor & & 9.7 & 19.57 & 21.57 & 34.63 \\
\hline \multirow[t]{3}{*}{$\mathrm{r}_{h}$} & pole & $0.3336 \pm 0.0014$ & $0.3277 \pm 0.0079$ & $0.3602 \pm 0.0115$ & $0.3581 \pm 0.0108$ \\
\hline & side & $0.3496 \pm 0.0017$ & $0.3425 \pm 0.0097$ & $0.3780 \pm 0.0142$ & $0.3751 \pm 0.0132$ \\
\hline & back & $0.3826 \pm 0.0025$ & $0.3725 \pm 0.0014$ & $0.4064 \pm 0.0203$ & $0.4012 \pm 0.0183$ \\
\hline \multirow[t]{3}{*}{$\mathrm{r}_{c}$} & pole & $0.3835 \pm 0.0013$ & $0.3777 \pm 0.0073$ & $0.3426 \pm 0.0115$ & $0.3338 \pm 0.0112$ \\
\hline & side & $0.4049 \pm 0.0017$ & $0.3977 \pm 0.0092$ & $0.3586 \pm 0.0142$ & $0.3485 \pm 0.0136$ \\
\hline & back & $0.4357 \pm 0.0024$ & $0.4260 \pm 0.0128$ & $0.3876 \pm 0.0213$ & $0.3751 \pm 0.0194$ \\
\hline $\mathrm{L}_{h}$ & & 0.4932 & 0.4485 & 0.5366 & 0.5356 \\
\hline $\mathrm{L}_{c}$ & & 0.5068 & 0.5516 & 0.4634 & 0.4144 \\
\hline$\sum(\mathrm{O}-\mathrm{C})^{2}$ & & 0.0081 & 0.0431 & 0.0194 & 0.0195 \\
\hline Spectral type & & K0-K2 & K0-K2 & G2-G0 & G2-G0 \\
\hline Co-Latitude $\left(^{\circ}\right)$ & & $57.29 \pm 1.21$ & $57.29 \pm 1.21$ & & \\
\hline Longitude $\left(^{\circ}\right)$ & & $259.29 \pm 3.28$ & $259.29 \pm 3.28$ & & \\
\hline Latitude $\left({ }^{\circ}\right)$ & & $11.45 \pm 0.42$ & $14.32 \pm 0.42$ & & \\
\hline $\mathrm{T}_{\text {spot }} / \mathrm{T}_{\text {local }}$ & & $0.40 \pm 0.035$ & $0.45 \pm 0.035$ & & \\
\hline
\end{tabular}

$$
\text { MinI }=2450622.558076(13)+0^{d} .37663(2) E
$$

The preliminary analysis was performed for a detached configuration, with no constraint on the potentials (Leung \& Wilson 1977), but the solution converged to variables showing a contact configuration. The gravity-darkening exponents were adopted to be $\mathrm{g}_{h}=\mathrm{g}_{c}=0.32$ for the convective envelopes (Lucy 1968), and the bolometric albedos were assumed to be $\mathrm{A}_{h}=\mathrm{A}_{c}=0.50$ (Rucinski 1969). In order to constrain the value of mass-ratio (q), inclination (i), and the mean surface temperature of the secondary component $T_{2}$, the non-dimensional surface potential $\Omega_{1}\left(=\Omega_{2}\right)$ and the monochromatic luminosity of the primary component $\mathrm{L}_{1}$ were taken as adjustable parameters. A q-search method was followed to constrain the mass ratio (q) and based on the least weight residuals, the mass ratio was freed to obtain the best-fit value. Our best solutions converged to q-values 1.30 and 0.81 for V1 and $\mathrm{V} 2$ in the I band, respectively. The final parameters obtained from the DC program were used in the light curve synthesis (LC) program to plot the synthetic light curves. The observed and synthetic light curves for both variables in I and V along with observed light curve in B band are shown in Fig. $1^{1}$. The solutions obtained are as listed in Table $2^{1}$.

\footnotetext{
${ }^{1}$ Table 1, Table 2 and Fig. 1 are from our recent publication Shanti Priya \& Rukmini (2017). For the meaning of different parameters listed in Table 2, we refer to Shanti Priya et al. (2013).
} 


\section{Discussion and Conclusions}

The photometric solutions (Table 2) in V and I bands for both the variables OGLE J003835.24735413.2 (V1) and OGLE J004619.65-725056.2 (V2) were obtained using the Wilson-Devinney method. The solutions for the I band indicate that V1 is a high-mass ratio shallow contact binary with $\mathrm{q} \simeq 1.3$ and fill-out factor or degree of contact (f) of $\sim 10 \%$. The light curve displays the O'Connell effect (Liu \& Yang 2003). The spot parameters are derived (see bottom part of Table 2). They indicate a cool spot on the primary component. Variable $\mathrm{V} 2$ has an inclination of $74^{\circ}$, with $\mathrm{q} \simeq 0.81$ and $\mathrm{f}$ $\simeq 22 \%$. The results indicate that both objects the are $\mathrm{W}$-type systems which are in poor geometrical (with low fill-out factor) and good thermal contact (low temperature difference between the components). The solutions also suggest that both variables are solar type marginal contact binaries. Such binaries play a key role in the understanding of the evolution from the near contact phase to the full contact phase (Liu et al., 2010). The 3.6-m Devasthal Optical Telescope (DOT) and the 4-m International Liquid Mirror Telescope (ILMT) operated by ARIES and located at the Devasthal Observaory can facilitate the continuous monitoring of such kind of objects which will help in understanding the reasons behind their period changes and to study their impact on cluster evolution. The complete study of these variable stars is presented by Shanti Priya \& Rukmini (2017).

\section{References}

Cox A.N. 2000, Allen's Astrophysical Quantities, Springer-Verlag, NY

Shanti Priya D., Rukmini J. 2017, AcAau, 134c, 303

Kwee K. K., Van Woerden H. 1956, BAN, 12, 327

Lenz P., Breger M. 2005, CoAst, 144, 41

Leung K. C., Wilson R. E. 1977, ApJ, 210, 853

Liu L., Qian S.-B., He J.-J. et al. 2010, PASJ, 62, 81

Lucy L. B. 1968, ApJ, 151, 1123

Mochnacki S. W. 1981, ApJ, 245, 650

Ruciński S. M. 1969, AcA, 19, 245

Shanti Priya D., Sriram K., Vivekananda Rao P. 2013, RAA, 13, 465

Stepien K. 2006, AcA, 56, 199

Udalski A., Kubiak M., Szymanski M. 1997, AcA, 47, 319

Udalski A. 1998, AcA., 48, 383

Van Hamme W., Wilson R. E. 2003, ASPC, 298, 323

Wilson R. E., Devinney E. J. 1971, ApJ, 166, 605

Wyrzykowski L., Udalski A., Kubiak M. et al. 2003, AcA, 53, 1

Yakut K., Eggleton P. P. 2005, ApJ, 629, 1055

Liu Q.-Y., Yang Y.-L. 2003, ChJAA, 3, 142 